Background: Cell based vaccines encoding Hyper-IL-6 (H6) and HyperIL-11 (H11) present high activity in murine melanoma and renal cancer model. We evaluated the efficacy of cellular vaccines modified with $\mathrm{H} 6$ or H11 combined with cyclophosphamide in orthotopic murine prostate cancer model.

Material and methods: TRAMP cells were transduced with $\mathrm{H} 6$ and $\mathrm{H} 11$ CDNA (TRAMP-H6 and TRAMP-H11). An orthotopic TRAMP model based on the implantation of TRAMP cells into the dorsolateral lobe of the prostate of C57BL6/J mice was employed. The efficacy of TRAMP-H6 and TRAMP-H11 vaccines evaluated in the therapeutic setting was compared with the TRAMP cells modified with a mock transduced E1-deleted adenoviral vector (TRAMP-AdV) and non-modified irradiated TRAMP cells (TRAMP IRR) in relation to naive (non-immunized) mice. In the next experimental groups mice vaccinated with TRAMP-H6 and TRAMP-H11 received cyclophosphamide (CY). Detection of immune cells in the spleen in mice receiving vaccines combined with $\mathrm{CY}$ was evaluated.

Results: Modification of TRAMP cells with $\mathrm{H} 6$ increased the efficacy of TRAMP-based whole-cell vaccine. The highest response rate was observed in mice receiving TRAMP-H6 alone and combined with CY. Vaccination with TRAMP-H6 alone and combined with $\mathrm{CY}$ and TRAMP $\mathrm{H} 11$ combined with $\mathrm{CY}$ extended median OS of mice bearing orthotopic TRAMP tumors in therapeutic setting. Low dose $\mathrm{CY}$ administered alone demonstrated some antitumor activity in employed model. TRAMP-H6 or TRAMP-H11 combined with $\mathrm{CY}$ strongly augmented generation of CD8+, CD4+ T lymphocytes and memory $T$ cells. Immunization with TRAMP combined with or without $C Y$ suppressed generation of $T$ regulatory cells.

Conslusions: Prostate cancer vaccines modified with $\mathrm{H} 6$ or $\mathrm{H} 11$ induce prostate tumour regression and increase mice survival by stimulating the immune system. Cyclophosphamide added to modified TRAMP vaccines demonstrated clinical benefit of treated mice and enhanced anti-tumour immune response.

Key words: cancer vaccine, immunotherapy, prostate cancer, cyclophosphamide, murine model.

Contemp Oncol (Pozn) 2015; 19 (3): 187-194 DOI: $10.5114 /$ wo.2015.52711

\section{Cell-based Hyper-interleukin 6 or Hyper-interleukin 11 secreting vaccines combined with low dose cyclophosphamide in an orthotopic murine prostate cancer model}

\author{
Jacek Mackiewicz ${ }^{1,2,3,4}$, Marek Kotlarski ${ }^{1}$, Ewelina Dondajewska ${ }^{1}$, \\ Anita Nowicka-Kotlarska ${ }^{1}$, Łukasz Krokowicz ${ }^{5}$, Urszula Kazimierczak ${ }^{1}$ \\ ${ }^{1}$ Chair of Medical Biotechnology, University of Medical Sciences, Poznan, Poland \\ 2Department of Diagnostics and Cancer Immunology, Greater Poland Cancer Centre, \\ Poznan, Poland \\ ${ }^{3}$ Department of Medical and Experimental Oncology, Clinical Hospital of Poznan \\ University of Medical Sciences, Poland \\ ${ }^{4}$ BioContract Sp. z o.o., Poznan, Poland \\ ${ }^{5}$ Chair and Department of General, Endocrinological and Gastroenterological, Oncology \\ Surgery, Poznan University of Medical Sciences, Poznan, Poland
}

\section{Introduction}

Sipuleucel- $T$ is to date the only approved therapeutic cancer vaccine. It has demonstrated survival benefit in advanced prostate cancer patients. Sipuleucel-T is an autologous dendritic cell (DC) vaccine loaded with prostate acid phosphatase fused with granulocyte-macrophage colony-stimulating factor (GM-CSF) [1]. Approval of Sipuleucel-T is the driving force for further development and evaluation of cancer vaccines in prostate cancer. Multiple immunotherapy strategies for prostate cancer are currently in preclinical and clinical studies, and a number of them have reached phase II and III [2, 3].

Irradiated whole cell based cancer vaccines deliver a wide spectrum of tumor antigens and induce anti-tumor specific immune responses [4-6]. Modification of vaccine cells with genes encoding immunostimulatory molecules can increase their immunogenicity. However, the effector function of vaccine-induced specific cytotoxic T-cells $(\mathrm{CTL})$ is inhibited by local tumor immune suppression mechanisms [7-10]. To enhance the efficacy of cancer vaccines, it might be crucial to combine them with strategies designed to subvert the tolerogenic processes in tumor-bearing hosts [11]. In order to enhance the anti-tumor immune response, therapeutic vaccination may require combination with other agents such as immune check-point inhibitors (anti-CTLA4, anti-PD1, anti-PDL-1 antibodies) or cytotoxic drugs. It was reported that cyclophosphamide (CY) administered prior to cancer vaccination is involved in non-specific blockade of immunosuppressive regulatory T lymphocytes (Tregs), enhancing the anti-tumor immune response [12, 13].

In the present study, we evaluated whether the addition of CY could enhance the therapeutic efficacy of whole cell based vaccines consisting of irradiated TRAMP cells modified with designer cytokines (Hyper-interleukin-6 [H6] or Hyper-interleukin-11 [H11]) in an orthotopic murine prostate cancer model. Hyper-interleukin-6 and $\mathrm{H} 11$ are artificial fusion cytokines composed of interleukin-6 (IL-6) or interleukin-11 (IL-11) linked by an artificial linker with corresponding soluble IL-6 receptor $\alpha$ (SIL-6R) or soluble IL11 receptor $\alpha(\mathrm{s} I L-11 \mathrm{R})$, respectively $[14,15]$. Both $\mathrm{H} 6$ and $\mathrm{H} 11$ directly bind to gp130 (signal transducing $\beta$ subunit of IL- 6 type cytokine receptor complex) and activate the JAK/STAT pathway. Although they bind the same cel- 
lular receptor subunit, they attract different domains of it, which may result in divergent biological activity [16]. Moreover, IL-6/SIL-6R complexes are detected in human serum and are functional. However, sIL-11R was not found in human body fluids so far; thus H11 type complexes (IL-11/sIL-11R) do not exist naturally in humans. Additionally, in an in vitro experimental model the binding of IL-11/ sIL-11R complex to gp130 was dependent on the density of the membrane-bound IL-11R.

It has previously been reported that gene-modified tumor vaccines secreting $\mathrm{H} 6$ or $\mathrm{H} 11$ displayed anti-tumor activity in an orthotopic murine renal cell cancer model [17, 18]. In the present study addition of CY to TRAMP-H6 and TRAMP-H11 vaccination enhanced generation of CD8+, CD4+ cytotoxic T lymphocytes and CD8+ and CD4+ memory T cells. TRAMP-H6 alone, TRAMP-H11 with $\mathrm{CY}$ and $\mathrm{CY}$ alone presented the highest clinical efficacy in mice bearing orthotopic TRAMP tumors. TRAMP vaccines administered with or without CY suppressed Tregs.

\section{Material and methods}

\section{Animals}

In vivo studies were conducted using 8-12 week old male C57BL6/J mice. The animals were purchased from the University of Medical Sciences (Lublin/Poland). Mice were housed and maintained under pathogen-free conditions in rooms with a 12-hour day/night cycle. All experiments were performed according to the guidelines approved by the Local Ethical Committee for Animal Research at the University of Medical Sciences (Poznań, Poland).

\section{Tumor cells}

Throughout the study a murine prostate cancer cell line (transgenic adenocarcinoma of mouse prostate TRAMP-C2) was used. TRAMP-C2 was purchased from the American Type Culture Collection and maintained in Dulbecco's modified Eagle's medium (DMEM) (Invitrogen Corp., Carlsbad, CA, USA) with 4 mM L-glutamine (Invitrogen Corp., Carlsbad, CA, USA) adjusted to contain $1.5 \mathrm{~g} / \mathrm{l}$ sodium bicarbonate (Life Technologies) and $4.5 \mathrm{~g} / \mathrm{l}$ glucose (Life Technologies) supplemented with $0.005 \mathrm{mg} / \mathrm{ml}$ bovine insulin (Gibco) and 5\% fetal bovine serum, 5\% NuSerum (Becton Dickinson), $10 \mathrm{nM}$ dehydroisoandrosterone (Life Technologies) and 5 gg/ml insulin (Life Technologies).

Two hundred ninety three (human embryonic kidney) cell line was used in in vitro experiments.

Cells were cultured on $78 \mathrm{~cm}^{2}$ culture plates at $37^{\circ} \mathrm{C}$ in a fully humidified atmosphere of $5 \% \mathrm{CO}_{2} / 95 \%$ air and passaged every 2-3 days.

\section{Adenoviral vectors modified with $\mathrm{H} 6$ and $\mathrm{H} 11$}

E1-deleted adenoviral recombinant vectors encoding $\mathrm{H} 6(\mathrm{AdH} 6)$ or $\mathrm{H} 11$ (AdH11) were constructed previously in the Department of Cancer Immunology at the University of Medical Sciences (Poznan, Poland). E1-deleted adenoviral vector (AdV) without a transgene (empty vector) was used as the control. The viruses were multiplied on E1-transfected 293 cells as described previously [19].

\section{Cancer vaccines}

Genetically modified whole cell based vaccines consist of TRAMP-C2 (TRAMP) cells transduced with cDNA-encoding H6 (TRAMP-H6) or H11 (TRAMP-H11). At 85\% confluence TRAMP cells were incubated with AdH6, AdH11 or AdV vector. After $48 \mathrm{~h}$, cells were harvested and subsequently irradiated with a dose of 80 Gy (Gamma-Cell 1000, RTA). Following irradiation, cells were stored in liquid nitrogen until use. A working cell bank was created (all experiments were conducted using single transduced cells).

\section{Chemotherapy}

Cyclophosphamide was purchased from Bristol-Myers Squibb and diluted in PBS for intraperitoneal injection (i.p.).

\section{Identification of $\mathrm{H} 6$ and $\mathrm{H} 11$ secreted by vaccine cells}

Irradiated TRAMP-H6 and TRAMP-H11 cells after thawing were cultured for $24 \mathrm{~h}$. Subsequently, in collected culture medium the concentration of accumulated $\mathrm{H} 6$ and H11 proteins was assessed with ELISA for human sIL-6R (R\&D) or human IL-11 (Anogen, Mississauga, Ontario, Canada). The quantities of both fusion proteins were calculated using SIL-6R or IL-11 protein standards, respectively. $\mathrm{H} 6$ is a fusion protein and consists of IL-6R, and therefore it reacts with anti-human sIL-6R antibody. Hyper-interleukin-11 is composed of IL-11 and reacts with anti-human IL-11 antibody.

\section{Orthotopic murine prostate cancer model}

To establish an orthotopic tumor model, mice underwent injection of TRAMP cells into the prostate. Prior to tumor cell administration, mice were anesthetized with i.p. injection of $0.7 \mathrm{mg} / \mathrm{g}$ Avertin. The subcutaneous tissue and abdominal muscles were cut with a scalpel, and by gently pulling the urinary bladder the prostate was exposed. Using a tuberculin syringe $2 \times 105$ TRAMP cells suspended in $20 \mu \mathrm{l}$ PBS were inoculated into the dorsolateral lobe of the prostate. Finally, the wound was closed with 4-5 surgical stitches.

The orthotopic murine prostate cancer rejection model was applied in the therapeutic setting. Mice were inoculated with TRAMP-WT cells into the prostate on day 0 . After $48 \mathrm{~h}$ mice were immunized (s.c. eight times in 4-day intervals) with TRAMP IRR, TRAMP-H6, TRAMP-H11, TRAMP-AdV. In the next experimental groups mice vaccinated with TRAMP-H6 and TRAMP-H11 received CY. The next group of mice received $\mathrm{CY}$ alone. The control group did not receive any treatment (untreated). Cyclophosphamide was administered i.p. in a dose of $50 \mathrm{mg} / \mathrm{kg}, 24 \mathrm{~h}$ before vaccination. The primary end-point of the experiment was overall survival (OS), which was assessed twice a week. All study groups comprised 10 animals.

In the second set of experiments mice were treated according to the same procedure as above and 12 weeks later were sacrificed. Prostate size was measured, and occurrence of lung metastases was assessed (macroscopic 
evaluation of lung tissue stained with dye ink). Tumor volume was calculated according to the formula $(\mathrm{W} 2 \times \mathrm{L} / 2)$, where $W$ is the shortest and $L$ the longest diameter.

\section{Detection of immune cells in the spleen}

Mice were immunized s.c. six times in 4-days intervals with TRAMP-H6, TRAMP-H11, TRAMP IRR with or without CY administered i.p. $24 \mathrm{~h}$ before each vaccination. An additional group received $C Y$ alone. Control mice were not vaccinated and did not receive $C Y$. Ten days after the last vaccination mice were sacrificed, and spleens were excised, minced and pooled (five per group). The number of splenocytes was counted after lysis of RBC. Splenocytes were stained with anti-CD4 (FITC), anti-CD8 (FITC) and anti-CD62L low (PE) for the detection of CD4+ and CD8+ memory $T$ lymphocytes, respectively. The single cell suspension was also stained with anti-CD25 (PE), anti-CD4 (FITC) and anti-FoxP3 (APC-Cy7) antibody for Tregs and anti-NK1.1 (PE) for detection of natural killer (NK) cells (Becton Dickin- son Biosciences). After acquisition, cells were analyzed on a FACSCanto cytometer (Becton Dickinson Biosciences).

\section{Results}

Irradiated TRAMP cells secrete $\mathrm{H} 6$ and $\mathrm{H} 11$ in vitro

Hyper-interleukin-6 and H11 fusion proteins were secreted by genetically modified TRAMP cells and detected in culture medium. The concentration of $\mathrm{H} 6$ and $\mathrm{H} 11$ was estimated to correspond to $1.5 \mathrm{\mu g} / \mathrm{ml} \mathrm{sIL-6R}$ and $0.08 \mathrm{\mu g} /$ $\mathrm{ml}$ IL-11, respectively (per $10^{6}$ cells $/ 24 \mathrm{~h}$ ).

High response rate and extended survival of treated mice in the orthotopic prostate cancer model

To evaluate the therapeutic effect of combining CY with murine prostate cancer vaccines modified with designer cytokines a set of experiments was conducted. After in-

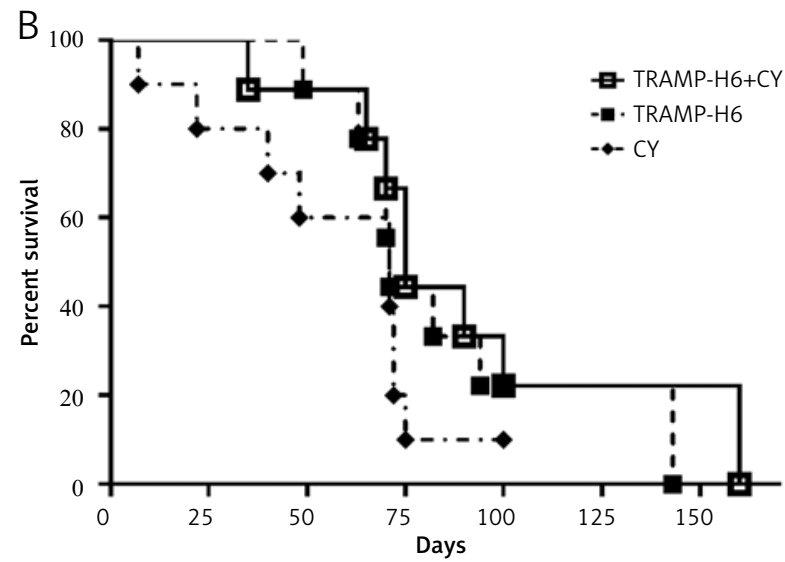

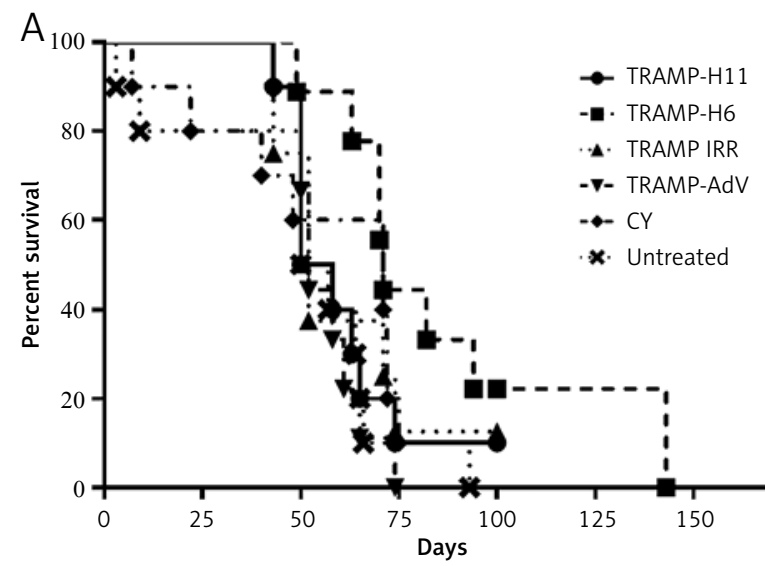

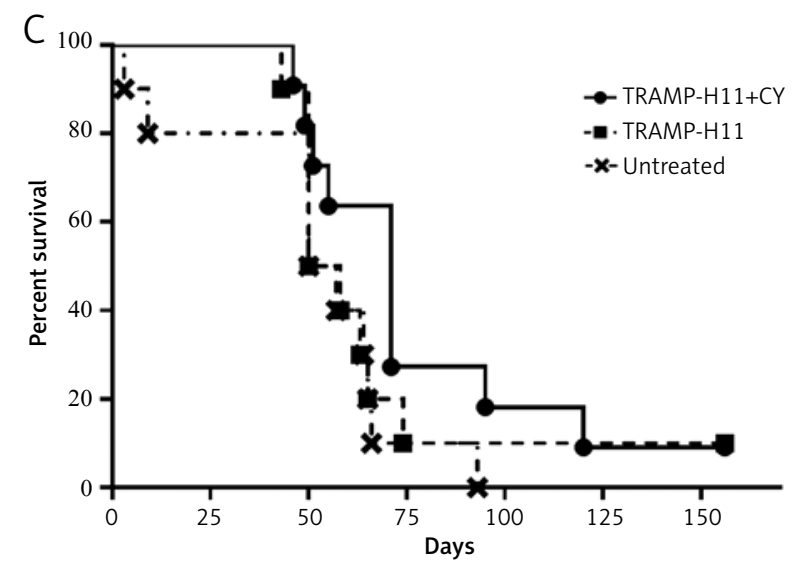

Fig. 1. Overall survival of mice treated in the orthotopic prostate cancer model. Mice were inoculated with TRAMP-WT cells into the prostate. Subsequently, the animals were vaccinated with TRAMP-H6 with or without cyclophosphamide, TRAMP-H11 with or without cyclophosphamide, TRAMP-AdV or TRAMP IRR. An additional group received cyclophosphamide alone. Control mice did not receive any treatment (untreated). A) Treatment with TRAMP-H6 elongated median OS compared to untreated controls $(p=0.01)$. There was a trend towards elongated median OS between TRAMP-H6 compared to TRAMP-H11, TRAMP IRR or TRAMP-AdV, but the differences were not statistically significant. Administration of $\mathrm{CY}$ alone was linked with longer survival compared to TRAMP-IRR, TRAMP-AdV, TRAMP-H11, and untreated control although the differences were not statistically significant. B) Mice receiving TRAMP-H6 + CY demonstrated the longest median OS. Combining TRAMP-H6 with $\mathrm{CY}$ was linked with elongation of median OS compared to TRAMP-H6 alone and CY alone, but the difference was not significant $(p=0.15$ and $p=0.34$, respectively). The median OS in the TRAMP-H6 + CY group was significantly longer compared to control mice $(p=0.01)$ (not shown in graph). C) Mice treated with TRAMP H11 + CY presented longer median OS compared to untreated controls $(p=0.03)$. Addition of $\mathrm{CY}$ to TRAMP-H11 compared to TRAMP-H11 elongated median OS of the treated mice but the difference was not statistically significant $(p=0.32)$ 


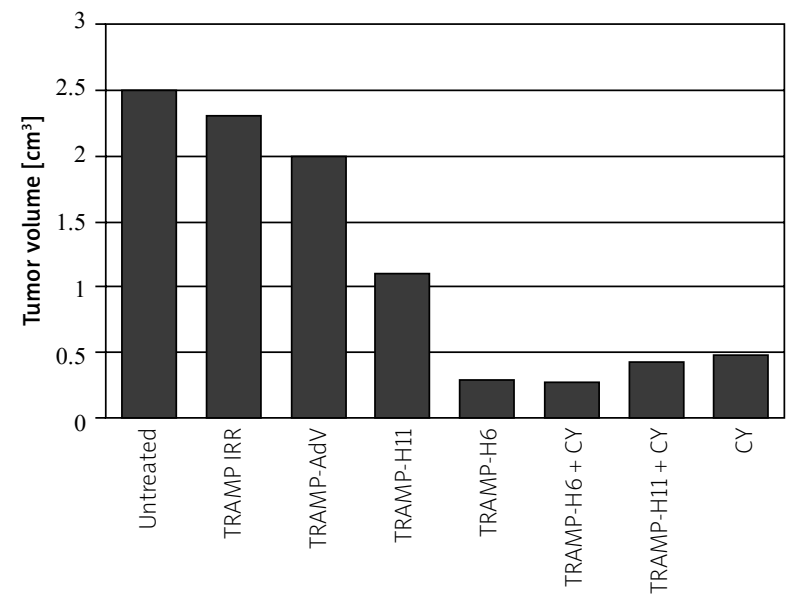

Fig. 2. Response assessment in murine prostate cancer model treated in the therapeutic setting. Twenty-four hours after inoculation of TRAMP-WT cells into the prostate mice were vaccinated with TRAMP-H6, TRAMP-H11, TRAMP-AdV or TRAMP IRR. Additionally, two groups received TRAMP-H6 and TRAMP-H11 combined with CY. One group was treated with $\mathrm{CY}$ alone. Control mice did not receive any treatment (untreated). Twelve weeks after the beginning of vaccination mice were sacrificed and tumor volume was assessed. The lowest mean tumor volume was observed in mice receiving TRAMP-H6, TRAMP-H6 + CY, TRAMP-H11, TRAMP-H11 + CY and CY alone

A
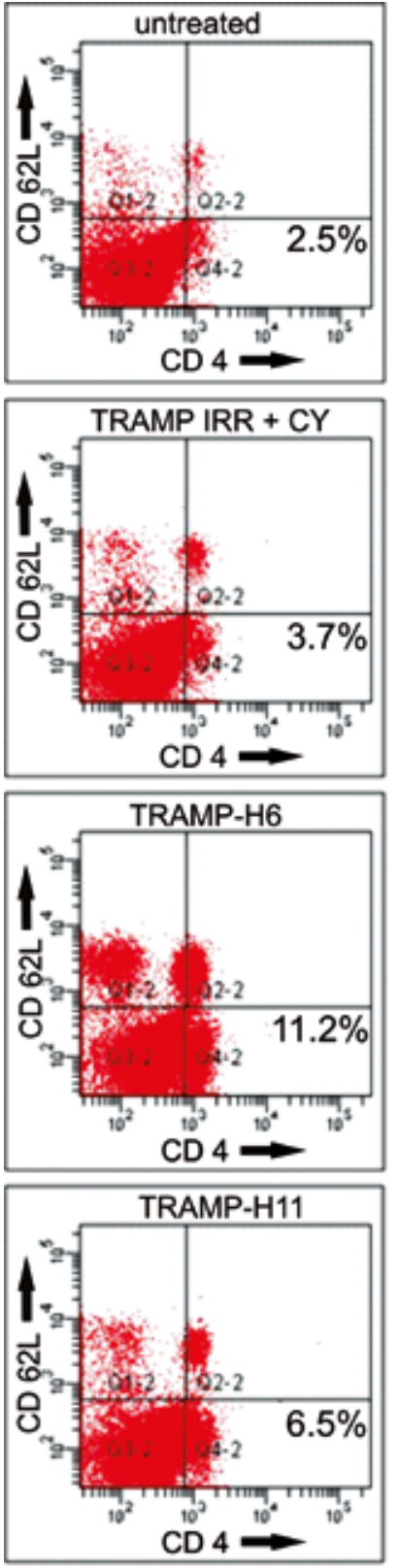
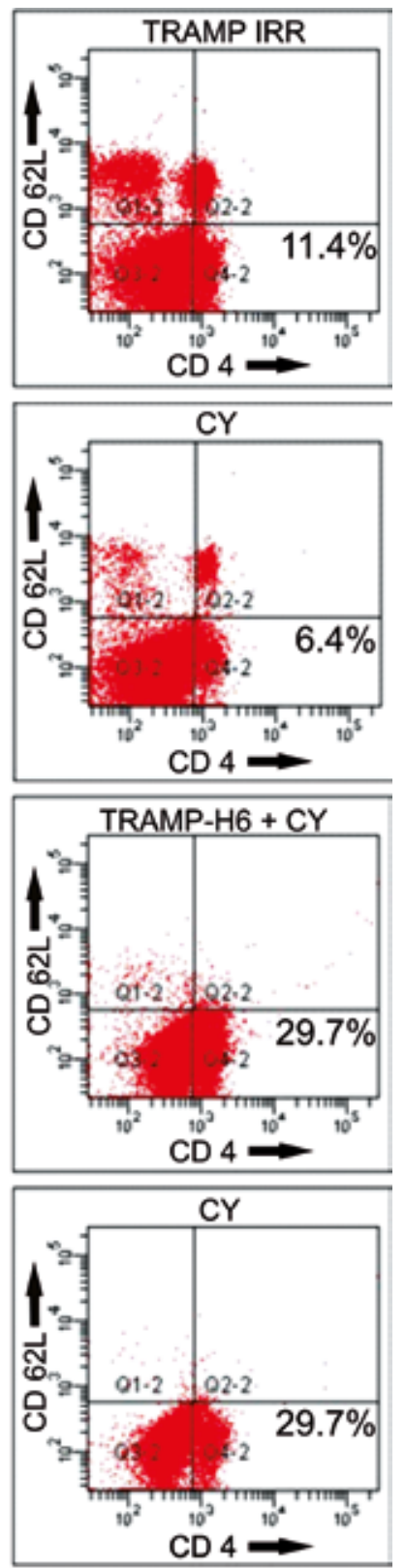

B
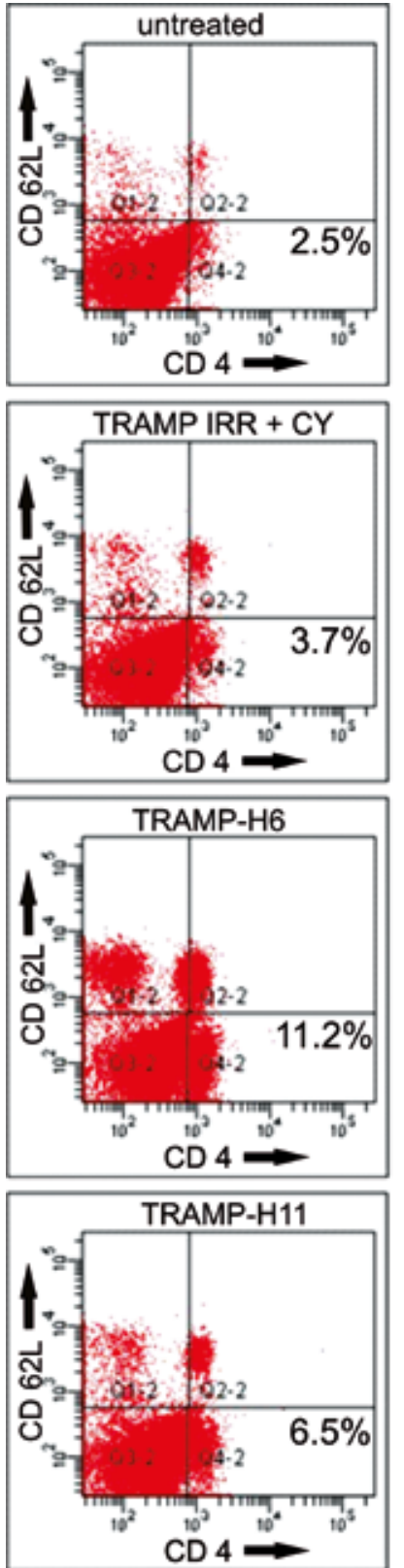
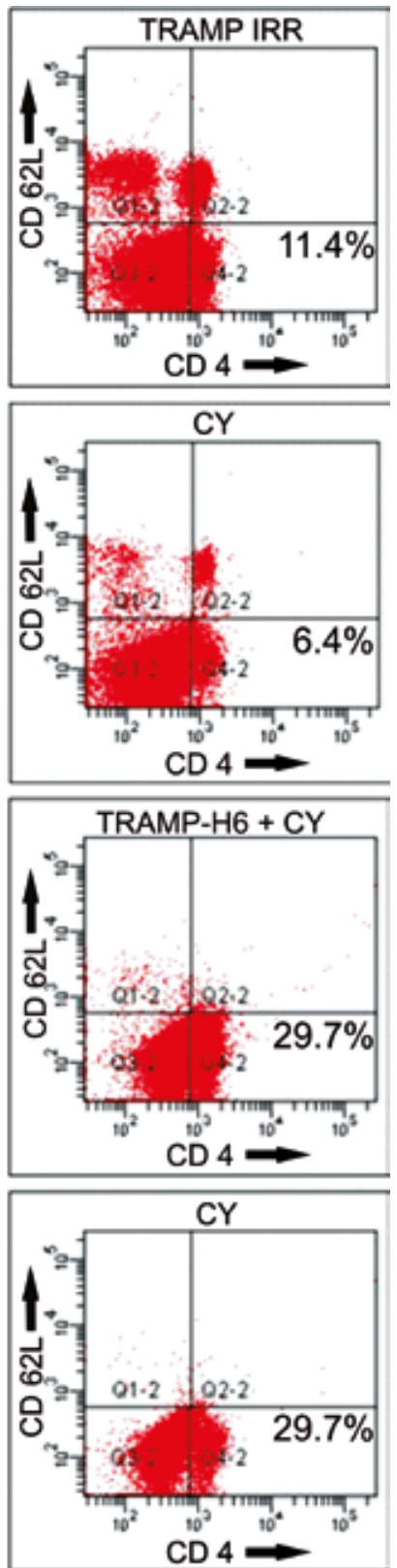

Fig. 3. Influence of repeated immunization (six times with 4-day interval) with TRAMP-H6, TRAMP-H11, TRAMP IRR with or without CY (administered i.p. $24 \mathrm{~h}$ before each vaccination) on memory T lymphocytes: A) CD8+ CD62L Low, B) CD4+ CD62 Low. An additional group received $\mathrm{CY}$ alone. Control mice were not vaccinated and did not receive $\mathrm{CY}$. Ten days after the last vaccination mice were sacrificed and spleens were excised, minced, pooled (five mice per group), stained and analyzed by flow cytometry 
oculation of TRAMP-WT cells into the prostate, mice were immunized with TRAMP-IRR, TRAMP-H6, TRAMP-H11, TRAMP-AdV, TRAMP-H6 + CY, TRAMP-H11 + CY or treated with $C Y$ alone. The control group did not receive any treatment (untreated).

Mice vaccinated with TRAMP-H6, TRAMP-H11, TRAMPAdV, TRAMP IRR or untreated controls demonstrated the following median OS - 71, 54, 52, 52 and 53 days, respectively (Fig. 1A). The combination of TRAMP-H6 with CY demonstrated the longest median OS (75 days) (Fig. 1B). The addition of CY to TRAMP-H11 was linked to longer median OS than TRAMP-H11 administered alone - 71 vs. 54 days (Fig. 1C). Mice receiving CY alone demonstrated a 72 day median OS (Fig. 1A, B).

To assess the effect of anti-tumor immune response, mice were treated according to the above procedure. TRAMP-WT cells were administered as above. Twelve weeks following vaccination, mice were sacrificed and tumor volume was assessed. All untreated mice developed prostate tumors (mean volume $2.5 \mathrm{~cm}^{3}$ ). Across all the remaining groups macroscopic prostate tumors were observed in 7-10 mice per group. The lowest mean tumor volume was observed in mice receiving TRAMP-H6 + CY $\left(0.28 \mathrm{~cm}^{3}\right)$, TRAMP-H6 $\left(0.30 \mathrm{~cm}^{3}\right)$, TRAMP-H11 + CY $(0.42$ $\left.\mathrm{cm}^{3}\right)$ and $\mathrm{CY}$ alone $\left(0.48 \mathrm{~cm}^{3}\right)$. In all remaining groups the mean volume was larger (TRAMP-H11 $-1.1 \mathrm{~cm}^{3}$, TRAMPAdV $-2.0 \mathrm{~cm}^{3}$, TRAMP IRR $\left.-2.2 \mathrm{~cm}^{3}\right)$. None of the mice developed lung metastases.

\section{Effect of vaccination with or without chemotherapy on immune response}

Studies were conducted on the effect of immunization with or without chemotherapy on individual immune cell subsets, using non-tumor bearing mice to rule out indirect effects of the drug on the immune system that result from reduced tumor size. After vaccination with TRAMP-H6, TRAMP-H11, TRAMP IRR with or without CY, mice were sacrificed and immune cells were analyzed. In an additional group CY alone was administered. Control mice did not receive any treatment.

\section{TRAMP-H6 or TRAMP-H11 combined with $\mathrm{CY}$ induces $\mathrm{CD} 4+$ and $\mathrm{CD} 8+\mathrm{T}$ lymphocyte responses}

The highest number of CD4+ and CD8+ T lymphocytes as well as memory $T$ cells (CD4+CD62L, CD8+CD62L) was observed in mice vaccinated with TRAMP-H6 combined with CY. Also a high percentage of the above cells was observed in animals receiving TRAMP-H11 + CY. Immunization with TRAMP-H11 vaccine showed the lowest impact on CD4+ and CD8+ T cells when compared to TRAMP-H6 $+\mathrm{CY}$, TRAMP-H11 + CY and TRAMP-H6 alone. However, the fraction of $C D 4+$ and $C D 8+T$ lymphocytes in mice vaccinated with TRAMP-H11 was still 2-3 times higher than in non-treated animals. CY alone is also able to induce an immune response with similar efficacy as TRAMP-H11. Interestingly, CY significantly enhanced the immune response of TRAMP cells modified with designer cytokines but weakened when administered with non-modified TRAMP cells (Fig. 3, Table 1).
Vaccines administered with or without CY did not attract NK cells.

\section{TRAMP vaccines with or without cyclophosphamide suppress $T$ regulatory lymphocytes}

Suppression of Tregs was noted in all study groups compared to non-vaccinated animals. The highest suppression of Tregs was observed in mice immunized with TRAMP-H11 + CY, TRAMP-H6 and TRAMP-IRR. Cyclophosphamide alone also decreased the Treg fraction. Interestingly, addition of CY to TRAMP-H11 decreased Tregs, but when combined with TRAMP-H6 the number of CD4+CD25+FoxP3+ cells was increased compared to mice receiving $C Y$ alone but still higher than in non-vaccinated animals (Fig. 4, Table 1).

\section{Discussion}

There are five major findings of these studies: (i) modification of TRAMP cells with the designer cytokine $\mathrm{H} 6$ increased the efficacy of TRAMP-based whole-cell vaccine; (ii) TRAMP-H6 alone and combined with CY and TRAMP $\mathrm{H} 11$ combined with CY extended median OS of mice bearing orthotopic TRAMP tumors in a therapeutic setting; (iii) combination of $\mathrm{CY}$ with TRAMP-H6 or TRAMP H11 strongly augmented generation of CD8+, CD4+ T lymphocytes and memory T cells (CD8+CD62L+, CD4+CD62L+); (iv) TRAMP vaccines combined with or without $C Y$ suppressed generation of Tregs; and (v) low dose CY administered alone demonstrated anti-tumor activity in the employed model.

All animals in the orthotopic murine prostate cancer model developed primary tumors with metastases to the regional lymph nodes. Metastases to lungs, bones or visceral organs were not observed (data not shown). Irradiated TRAMP cells transduced with $\mathrm{H} 6$ or $\mathrm{H} 11$ served as therapeutic vaccines in the prostate cancer model. Our earlier studies demonstrated that whole cell vaccines modified with $\mathrm{H} 6$ and $\mathrm{H} 11$ are very effective in inducing anti-tumor specific immune responses and extension of survival of animals in a murine orthotopic renal cell carcinoma (RENCA) model $[17,18]$. Moreover, $\mathrm{H} 6$ modified human therapeutic whole cell melanoma vaccine has shown patient benefits in clinical trials [20, 21].

Generation of a specific antitumor immune response is a multistep process, involving DCs, NK cells, CD8+ and CD4+ T cells. Dendritic cells are responsible for processing and presentation of cancer antigens to $T$ lymphocytes in the context of the major histocompatibility complex. At the site of vaccination designer cytokines ( $\mathrm{H} 6$ and $\mathrm{H} 11$ ) activated maturation of DCs and thus increased tumor antigen presentation $[17,18]$. Moreover, $\mathrm{H} 6$ secreted at the vaccination site stimulated presentation of cryptic antigens by DCs, broadening the spectrum of target antigens recognized by effector T cells [17]. It has also been shown that $\mathrm{H} 6$ and $\mathrm{H} 11$ secreted by irradiated RENCA cells at the vaccine site reduced the infiltration by Tregs $[17,18]$, which may directly suppress activation and maturation of DCs [22]. After induction of the immune response, activated antigen-specific cytotoxic, helper T-cells, B- lymphocytes and NK cells enter the effector phase. In our earlier studies 

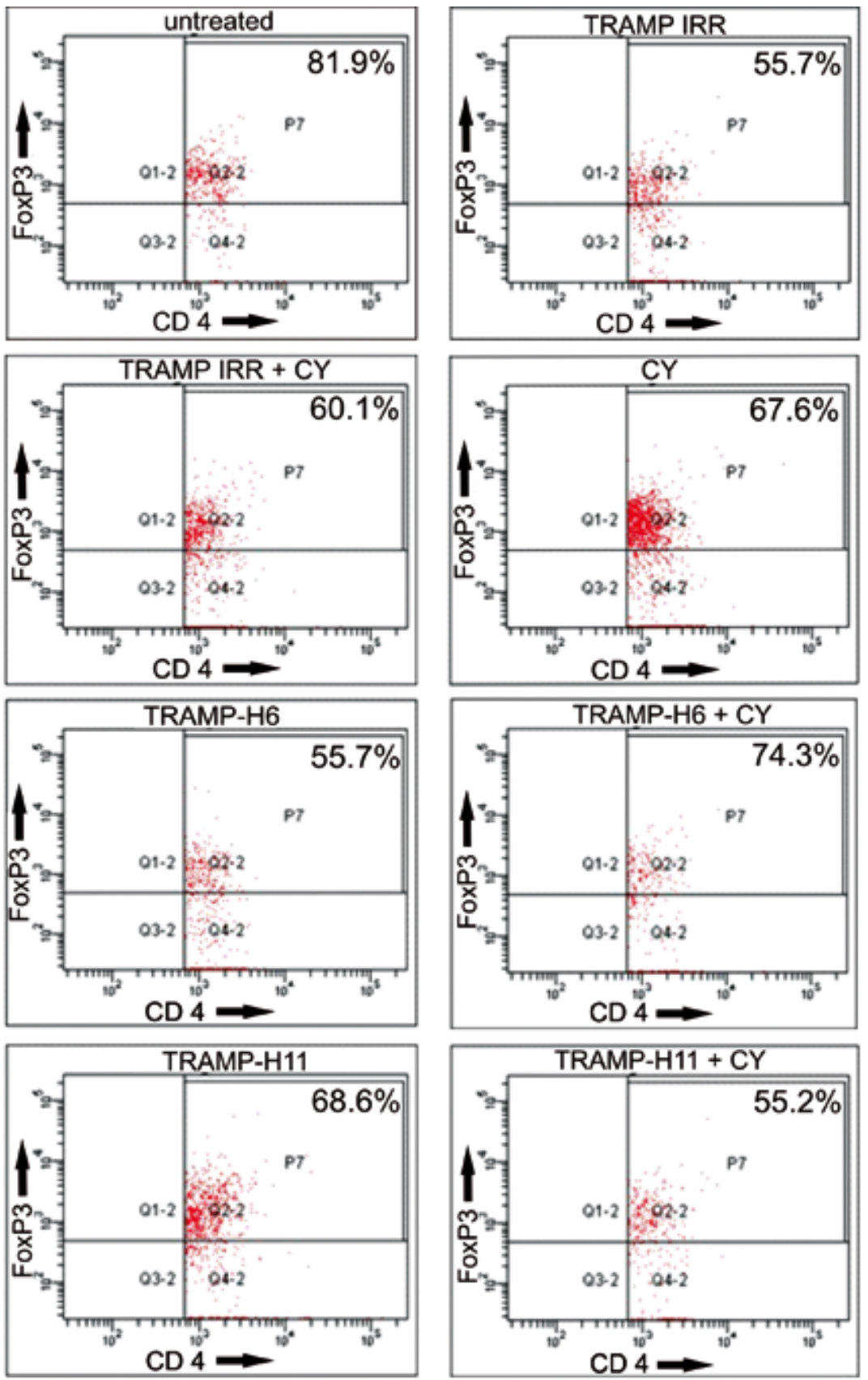

Fig. 4. Flow cytometric analyses of $\mathrm{T}$ regulatory lymphocytes (CD4+CD25+FoxP3+) assessed in the spleen after vaccination with TRAMP-H6, TRAMP-H11, TRAMP IRR with or without CY (administered i.p. $24 \mathrm{~h}$ before each vaccination). An additional group received CY alone. Control mice were not vaccinated and did not receive $\mathrm{CY}$

in murine melanoma and RENCA models, we found that vaccination with $\mathrm{H6}$-modified cells resulted in generation of antigen-specific CD8+, CD4+ T lymphocytes [17, 23]. Finally, effector cells reach the tumor site and should eliminate cancer cells. In mice vaccinated with RENCA-H6 and RENCA-H11, high infiltration of tumors by activated CD8+, CD4+ T cells and NK cells was observed $[17,18]$.

In the present study we used the alkylating agent $C Y$ to enhance the anti-tumor immune response induced by therapeutic cancer vaccination. It has been reported that $\mathrm{CY}$ in low doses combined with cancer vaccines enhanced depletion of immunosuppressive Tregs and activation of antigen-specific CD8+ and CD4+ T cells [13, 24-27]. We found that TRAMP-H6 vaccine significantly reduced tumor volume and extended OS of treated mice compared to untreated controls. Our previous studies have also shown that RENCA-H6 vaccine demonstrated high therapeutic efficacy in renal carcinoma [17]. Mice treated with TRAMP-H6 combined with CY demonstrated the longest median OS. Animals receiving TRAMP-H11 with $\mathrm{CY}$ demonstrated identical median OS as animals receiving TRAMP-H6 and $\mathrm{CY}$ alone. Low dose CY administered alone is able to reduce tumor volume in a TRAMP orthotopic model, although the median OS in mice receiving CY alone compared to 
Table 1. Effect of immunization with or without CY on splenic cell populations. C57BL/6 mice ( $n=5$ per group) were vaccinated with s.c. six times in 3-day interval with TRAMP-H6, TRAMP-H11, TRAMP IRR with or without CY (administered i.p. $24 \mathrm{~h}$ before each vaccination). Additional group received CY alone. Control mice were not vaccinated and did not receive CY. After lysis of RBC, splenocytes were counted and stained for T cells (CD4+, CD8+, CD62 Low), NK cells (NK1.1) and Tregs (CD4+CD25+FoxP3+). Cells were analysed by flow cytometry

\begin{tabular}{|c|c|c|c|c|c|c|}
\hline \multirow[t]{2}{*}{ Immunization } & \multicolumn{6}{|c|}{ Population } \\
\hline & $\begin{array}{l}\text { CD8+ } \\
(\%)\end{array}$ & $\begin{array}{l}\text { CD4+ } \\
(\%)\end{array}$ & $\begin{array}{l}\mathrm{CD} 8+\mathrm{CD} 62 \mathrm{~L} \\
(\%)\end{array}$ & $\begin{array}{l}\mathrm{CD} 4+\mathrm{CD} 62 \mathrm{~L} \\
(\%)\end{array}$ & $\begin{array}{l}\mathrm{NK} \\
\text { (\%) }\end{array}$ & $\begin{array}{c}\mathrm{CD} 4+\mathrm{CD} 25+\mathrm{FoxP3} \\
\text { (\%) }\end{array}$ \\
\hline TRAMP-H6 & 10.8 & 18.9 & 4.8 & 11.2 & 2.6 & 55.7 \\
\hline TRAMP-H6 CY & 38.2 & 30.1 & 36.7 & 29.7 & 6.3 & 74.3 \\
\hline TRAMP-H11 & 3.1 & 8.9 & 1.6 & 6.5 & 5.4 & 68.6 \\
\hline TRAMP-H11 CY & 25.5 & 22.4 & 24.2 & 21.9 & 3.9 & 55.2 \\
\hline TRAMP IRR & 9.2 & 17.5 & 4.2 & 11.4 & 2.5 & 55.7 \\
\hline TRAMP IRR CY & 2.2 & 5.2 & 1.1 & 3.7 & 6.8 & 60.1 \\
\hline CY & 24.3 & 8.9 & 19.9 & 6.4 & 5.8 & 67.6 \\
\hline Non-vaccinated & 1.3 & 3.1 & 0.6 & 2.5 & 3.9 & 81.9 \\
\hline
\end{tabular}

non-treated animals was not statistically different. We observed that CY administered alone was able to reduce Tregs and enhance generation of CD8+ T lymphocytes and CD8+ memory $T$ cells in the spleen. A trend toward a therapeutic effect of $\mathrm{CY}$ low doses was also reported by others [13]. Accordingly, some additive mechanisms are possible.

In a recently presented study, addition of low dose CY (50 mg/mice) to whole cell vaccine secreting GM-CSF (GVAX) in a murine hepatocellular model enhanced the anti-tumor effect of the vaccine. It was found that GVAX with $C Y$ induced the highest number of antigen-specific CD8+ and CD4+ in the spleen and at the tumor site. Moreover, mice treated with GVAX and CY presented the lowest number of tumor-infiltrating Tregs [27]. We found that $\mathrm{CY}$ added to TRAMP-H6 or TRAMP-H11 vaccine enhanced the efficacy of vaccines and induced generation of CD8+, CD4+ T lymphocytes and CD8+, CD4+ memory $T$ cells and reduced Tregs in the spleen.

The results of this study showed that both TRAMP-H6 and TRAMP-H11 vaccines when combined with $\mathrm{CY}$ generated the strongest stimulation of the immune response. Moreover, TRAMP H6 and TRAMP-H11 administered with $\mathrm{CY}$ induced the highest tumor regression and longest OS of treated animals.

The authors declare no conflict of interest.

The study was supported by a grant from the Ministry of Science and Higher Education, Warsaw, Poland (No. N N401582038).

\section{References}

1. Kantoff PW, Higano CS, Shore ND, et al. Sipuleucel-T immunotherapy for castration-resistant prostate cancer. N Engl J Med 2010; 363: 411-22.

2. Fong L, Small EJ. Immunotherapy for prostate cancer. Curr Oncol Rep 2007; 9: 226-33.

3. Heidegger I, Massoner P, Eder IE, et al. Novel therapeutic approaches for the treatment of castration-resistant prostate cancer. J Steroid Biochem Mol Biol 2013; 138: 248-56.

4. Mach N, Dranoff G. Cytokine-secreting tumor cell vacines. Curr Opin Immunol 2000; 12: 571-5.
5. Ward S, Casey D, Labarthe MC, Whelan M, Dalgleish A, Pandha H, Todryk S. Immunotherapeutic potential of whole tumor cells. Cancer Immunol Immunother 2002; 51: 351-357.

6. Kozłowska A, Mackiewicz J, Mackiewicz A. Therapeutic gene modified cell based cancer vaccines. Gene 2013; 525: 200-7.

7. Thomas AM, Santarsiero LM, Lutz ER, et al. Mesothelin-specific CD8(+) T cell responses provide evidence of in vivo cross-priming by antigen-presenting cells in vaccinated pancreatic cancer patients. J Exp Med 2004; 200: 297-306.

8. Bronte V, Kasic T, Gri G, et al. Boosting antitumor responses of $T$ lymphocytes infiltrating human prostate cancers. J Exp Med 2005; 201: 1257-68.

9. Gajewski TF. Failure at the effector phase: immune barriers at the level of the melanoma tumor microenvironment. Clin Cancer Res 2007; 13: 5256-61.

10. Drake CG, Jaffee E, Pardoll DM. Mechanisms of immune evasion by tumors. Adv Immunol 2006; 90: 51-81.

11. Pardoll D, Allison J. Cancer immunotherapy: breaking the barriers to harvest the crop. Nat Med 2004; 10: 887-92.

12. Liu JY, Wu Y, Zhang XS, et al. Single administration of low dose cyclophosphamide augments the antitumor effect of dendritic cell vaccine. Cancer Immunol Immunother 2007; 56: 1597-1604.

13. Wada S, Yoshimura K, Hipkiss EL, et al. Cyclophosphamide augments antitumor immunity: studies in an autochthonous prostate cancer model. Cancer Res 2009; 69: 4309-18.

14. Peters M, Blinn G, Solem F, Fischer M, Meyer zum Büschenfelde $\mathrm{KH}$, Rose-John S. In vivo and in vitro activities of the gp130-stimulating designer cytokine Hyper-IL-6. J Immunol 1998; 161: 3575-81.

15. Mackiewicz A, Dams-Kozlowska H, Rose-John S. Chimeric soluble Hyper-IL-11 and use thereof. Patent No WO/2005/113591. 2005.

16. Kurth I, Horsten U, Pflanz S, Dahmen H, Küster A, Grötzinger J, Heinrich PC, Müller-Newen G. Activation of the signal transducer glycoprotein 130 by both IL- 6 and IL-11 requires two distinct binding epitopes. J Immunol 1999; 162: 1480-7.

17. Wysocki PJ, Kazimierczak U, Suchorska W, Kotlarski M, Malicki J, Mackiewicz A. Gene-modified tumor vaccine secreting a designer cytokine Hyper-Interleukin-6 is an effective therapy in mice bearing orthotopic renal cell cancer. Cancer Gene Ther 2010; 17: 465-75.

18. Suchorska WM, Dams-Kozlowska H, Kazimierczak U, Wysocki PJ, Mackiewicz A. Hyper-interleukin-11 novel designer molecular adjuvant targeting gp130 for whole cell cancer vaccines. Expert Opin Biol Ther 2011; 11: 1555-67.

19. Kowalczyk DW, Wlazlo AP, Shane S, Ertl HC. Vaccine regimen for prevention of sexually transmitted infections with human papillomavirus type 16. Vaccine 2001; 19: 3583-90.

20. Mackiewicz A, Mackiewicz J, Wysocki PJ, et al. Long-term survival of high-risk melanoma patients immunized with a Hyper-IL-6-modified allogeneic whole-cell vaccine after complete resection. Expert Opin Investig Drugs 2012; 21: 773-783. 
21. Mackiewicz J, Karczewska-Dzionk A, Laciak M, et al. Whole cell therapeutic vaccine modified with Hyper-IL6 for combinational treatment of non-resected advanced melanoma. Medicine 2015 (in press).

22. Shevach EM. CD4+CD25+ suppressor T cells: more questions than answers. Nat Rev Immunol 2002; 2: 389-400.

23. Izycki D, Nawrocki S, Laciak M, Gryska K, Baksalary-Izycka K, Mackiewicz A. Effect of cyclophosphamide on tumorogenesis of IL-6 and Hyper-IL-6 gene modified murine melanoma cells. Contemp Oncol 2004; 8: 124-31.

24. Machiels JP, Reilly RT, Emens LA, Ercolini AM, Lei RY, Weintraub D, Okoye FI, Jaffee EM. Cyclophosphamide, doxorubicin, and paclitaxel enhance the antitumor immune response of granulocyte/ macrophage-colony stimulating factor-secreting whole-cell vaccines in HER-2/neu tolerized mice. Cancer Res 2001; 61: 36893697.

25. von Boehmer H. Mechanisms of suppression by suppressor T cells. Nat Immunol 2005; 6: 338-44.

26. Sakaguchi S, Yamaguchi T, Nomura T, Ono M. Regulatory T cells and immune tolerance. Cell 2008; 133: 775-87.

27. Chen C, Hou J, Lin Z, et al. A bystander cell-based GM-CSF secreting vaccine synergized with a low dose cyclophosphamide presents therapeutic immune response against murine hepatocellular carcinoma. Cell Mol Immunol 2013; 10: 349-59.

\section{Address for correspondence}

\section{Jacek Mackiewicz}

Department of Cancer Immunology

Chair of Medical Biotechnology

Poznan University of Medical Sciences

Greater Poland Cancer Centre

Garbary 15

61-866 Poznan

Submitted: 27.04 .2015

Accepted: 27.05 .2015 